\title{
A Novel Power Allocation Scheme under Outage Constraints in NOMA Systems
}

\author{
Jingjing Cui, Student Member, IEEE, Zhiguo Ding, Senior Member, IEEE, Pingzhi Fan, Fellow, IEEE
}

\begin{abstract}
In this letter, we study a downlink non-orthogonal multiple access (NOMA) transmission system, where only average channel state information is available at the transmitter. Two criteria - transmit power and fairness for NOMA systems are studied, subjected to probabilistic constraints on the rate outage. We develop the optimal power allocation scheme in closed-form by employing the feature of the NOMA principle. Furthermore, the power difference between NOMA systems and OMA systems under outage constraints is attained. Finally, the provided simulation results demonstrate that NOMA outperforms OMA with the two proposed power allocation schemes.

Index Terms-Non-orthogonal multiple access (NOMA), average channel state information, power allocation, probabilistic constraints
\end{abstract}

\section{INTRODUCTION}

Recently, non-orthogonal multiple access (NOMA) based on successive interference cancellation (SIC) has been recognized as one of the promising multiple access technologies to be used for wireless communication towards 2020 and beyond [1]. Different from conventional orthogonal multiple access (OMA), e.g. time-division multiple access (TDMA), NOMA introduces an new dimension - power domain for multiple access. Therefore, the NOMA system can adjust the number of connections and the quality of service (QoS) of users by allocating different power levels to different users. In this case, users' power allocation becomes an important issue in designing a NOMA system [2]-[4].

The works in [3] and [4] focused on analysing the performance of the NOMA scheme in terms of outage probability and user paring, where the fixed power allocation scheme is characterized. To further employ the potential gain of NOMA in the power domain, the problem to maximize the worst user throughput with the total throughput constraint has been proposed in [2] and a similar result has been conducted in [5] with transmit power constraints. Most of the existing works on NOMA assume that perfect channel state information (CSI) is available at the transmitter. However, such an assumption is considered idealistic [6] due to the limited CSI feedback. In general, imperfect CSI can lead to substantial performance degradation, such as QoS outages, if not taken care of properly. In [5] and [7], outage balancing among users has been studied separately under average CSI, where the fairness of the weak users has been considered with dynamic decoding order and user pair separately.

J. Cui and P. Fan are with the Institute of Mobile Communications, Southwest Jiaotong University, Chengdu 610031, P. R. China. (email: cuijingj@foxmail.com,p.fan@ieee.org).

Z. Ding is with the School of Computing and Communications, Lancaster University, LA1 4YW, UK. (e-mail: z.ding@lancaster.ac.uk).
This paper studies transmit power minimization and fairness for the system under a probabilistic outage constraint for a multi-user downlink NOMA system, where perfect CSI is known at receivers and only average CSI is available at the base station (BS), i.e., only the location information of the users is known to the BS. We assume that the users are static and consider the SIC decoding order based on the distances between users and the BS. Specially, an optimal power allocation scheme is derived in a closed-form expression under the outage constraint in NOMA systems. In addition, comparisons between the NOMA scheme and the conventional OMA scheme is conducted from the power consumption with the outage constraint. The results conclude that the NOMA scheme outperforms the conventional OMA scheme from the perspective of power consumption and fairness.

\section{SySTEM MODEL}

Consider a cellular downlink NOMA scenario with one BS and $M$ users. All nodes are equipped with a single antenna. The NOMA principle is used for transmission purposes. Therefore, the observation at the $m$-th user is given by

$$
y_{m}=h_{m} \sum_{i=1}^{M} \sqrt{P_{i}} s_{i}+n_{m},
$$

where $s_{i}$ is the message for the $m$-th user, $h_{m}$ denotes the channel gain between the $\mathrm{BS}$ and the $m$-th user. We assume perfect CSI is available on the users, but only average CSI is available on the BS. In particular, it is assumed that $h_{m}=$ $d_{m}^{-\gamma} g_{m}$, with $d_{m}$ being the distance from the $m$-th user to the BS, where $\gamma$ is the pass loss exponent, and $g_{m} \sim \mathcal{C N}(0,1)$. Without loss of generality, the distances are sorted as $d_{1} \geq$ $d_{2} \geq \cdots \geq d_{M} . P_{m}$ is the transmission power allocated for the $m$-th user and $n_{m}$ denotes zero-mean additive noise of the $m$-th user with variance $\sigma^{2}$.

In this case, each user employs SIC in an successive order. Let $R_{j}^{m}$ denote the rate for the $m$-th user to detect the $j$-th user's message, $j \leq m$, which can be expressed as

$$
R_{j}^{m}=\log \left(1+\frac{\left|h_{m}\right|^{2} P_{j}}{\sum_{i=j+1}^{M}\left|h_{m}\right|^{2} P_{i}+\sigma^{2}}\right) .
$$

Since the perfect CSI is not available at the BS, an outage event may happen in NOMA systems, which is defined as that the $m$-th user is not able to decode its own message or the message of the weaker user $j<m$ [3].

Therefore, the outage probability at the $m$-th user can be expressed as

$$
\begin{aligned}
P_{m}^{\text {out }} & =1-P\left(R_{1}^{m} \geq \tilde{R}_{1}, \cdots, R_{m}^{m} \geq \tilde{R}_{m}\right) \\
& =1-e^{-\lambda_{m} \max _{j=1, \cdots, m} \frac{\phi_{j} \sigma^{2}}{P_{j}-\phi_{j} \sum_{i=j+1}^{M} P_{i}},}
\end{aligned}
$$

where $\lambda_{m}=d_{m}^{\gamma}, \phi_{j}=2^{\tilde{R}_{j}}-1$ and $\tilde{R}_{j}$ is the targeted data 
rate of the $j$-th user. Note that $P_{j}>\phi_{j} \sum_{i=j+1}^{M} P_{i}$. If $P_{j} \leq$ $\phi_{j} \sum_{i=j+1}^{M} P_{i}$, the outage probability of the $j$-th user will be always one.

\section{Problem formulation}

In NOMA, the power allocation is important to the achievable rate of each user due to power-domain multi-user multiplexing. In this section, two power allocation schemes based on two criteria-transmit power and fairness will be studied separately where only average CSI is known at the BS.

\section{A. Minimizing total transmission power}

The problem of interest here is to design the system, which should provide an acceptable QoS requiring as little transmit power as possible, and serving as many users as possible. In particular, the problem of minimizing the total transmit power subjected to individual outage constraints can be formulated as follows:

$$
\begin{aligned}
\min _{\left\{P_{m}\right\}} & \sum_{m=1}^{M} P_{m} \\
\text { s.t. } & P_{m}^{\text {out }} \leq \epsilon_{m}, \quad \forall m, \\
& P_{m}>\phi_{m} \sum_{i=m+1}^{M} P_{i}, m=1 \cdots, M, \\
& P_{m} \geq 0, \quad \forall m .
\end{aligned}
$$

Specifically, $\epsilon_{m} \in[0,1)$ is denoted by the maximum tolerable outage probability for the $m$-th user. The constraint in (4b) can be transformed to be a linear constraint as follows:

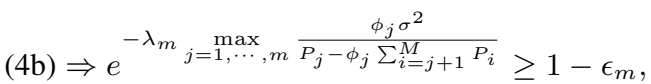

$$
\begin{aligned}
& \Rightarrow \max _{j=1, \cdots, m} \frac{\phi_{j} \sigma^{2}}{P_{j}-\phi_{j} \sum_{i=j+1}^{M} P_{i}} \leq \frac{1}{\lambda_{m}} \ln \frac{1}{1-\epsilon_{m}}, \\
& \Rightarrow \min _{j=1, \cdots, m} \frac{P_{j}-\phi_{j} \sum_{i=j+1}^{M} P_{i}}{\phi_{j}} \geq \frac{\lambda_{m} \sigma^{2}}{\ln \frac{1}{1-\epsilon_{m}}},
\end{aligned}
$$

where the basic properties of inequalities are used to obtain $(5 b)$ and $(5 c)$. In $(5 b)$, the $\ln (\cdot)$ operator is used for both side, since $\ln (\cdot)$ does not affect the inequality. Therefore, (4b) has been transformed into a linear constraint. The closed form expression of the optimal solution for the addressed optimization problem can be obtained in the following. To derive a closed-form expression of power allocation, we first introduce the following Proposition.

Proposition 1: Let $x_{m}=P_{m}-\phi_{j} \sum_{i=j+1}^{M} P_{i}, 1 \leq j \leq$ $M-1$, and $x_{M}=P_{M}$ with $x_{m}>0, \forall m$. The equations in (8) at the top of next page are guaranteed.

Proof: We introduce the auxiliary $x_{m}, x_{m}>0, \forall m$, with $x_{m}=P_{m}-\phi_{j} \sum_{i=j+1}^{M} P_{i}, 1 \leq j \leq M-1$, and $x_{M}=P_{M}$. For any $i^{\prime}, P_{M-i^{\prime}}$ is given by

$$
\begin{aligned}
P_{M-i^{\prime}}= & x_{M-i^{\prime}}+ \\
& \phi_{M-i^{\prime}}\left[x_{M-i^{\prime}+1}+\sum_{k=2}^{i^{\prime}} \prod_{j=1}^{k-1}\left(1+\phi_{M-i^{\prime}+j}\right) x_{M-i^{\prime}+k}\right] .
\end{aligned}
$$

Let $m=M-i^{\prime}$, it's easy to obtain (8). In addition, the total power

$$
\sum_{m=1}^{M} P_{m}=\sum_{m=1}^{M}\left(x_{m}+\phi_{m}\left(x_{m+1}+\prod_{i=m+1}^{M-1}\left(1+\phi_{i}\right) x_{i+1}\right)\right),
$$

which can be calculated in (8b) with some manipulations.

From (8b), the minimization transmit power problem in (4) can be reformulated as

$$
\begin{array}{ll}
\min _{\left\{x_{m}\right\}} & \sum_{m=1}^{M} \beta_{m} x_{m} \\
\text { s.t. } & \min _{j=1, \cdots, m} \frac{x_{j}}{\phi_{j}} \geq \frac{\lambda_{m} \sigma^{2}}{\log \frac{1}{1-\epsilon_{m}}}, m=1, \cdots, M, \\
& x_{m}>0, \quad \forall m .
\end{array}
$$

To derive the solution in closed-form for (10), we introduce Proposition 2.

Proposition 2: The optimal solution for problem (10) is given by:

$$
x_{m}=\phi_{m} \max \left\{\frac{\lambda_{m} \sigma^{2}}{\log \frac{1}{1-\epsilon_{m}}}, \cdots, \frac{\lambda_{M} \sigma^{2}}{\log \frac{1}{1-\epsilon_{M}}}\right\}, \quad \forall m .
$$

Specially, if $\epsilon_{m}=\epsilon, \forall m$, then $x_{m}=\phi_{m} \frac{\lambda_{m} \sigma^{2}}{\log \frac{1}{1-\epsilon_{m}}}, \forall m$.

Proof: For any $j$ with $1 \leq j \leq M$, the first inequality in (10b) can be equivalently expressed as

$$
\frac{x_{j}}{\phi_{j}} \geq \max \left\{\frac{\lambda_{j} \sigma^{2}}{\log \frac{1}{1-\epsilon_{j}}}, \cdots, \frac{\lambda_{M} \sigma^{2}}{\log \frac{1}{1-\epsilon_{M}}}\right\} .
$$

It is obvious that $x_{1}, \cdots, x_{M}$ are independent and the objective function of (10) is linear. Therefore, the optimal solution of (10) will be obtained at all the constraints with equalities. The intuition behind Proposition 2 is that all $P_{m}, \forall m$, must be positive for the problem constraints to be satisfied, while all $\tilde{R}_{j}$ must be equal to the minimum of $R_{j}^{m}, \forall m$ with $m \geq j$. This is based on the fact that the decoding order is constant because the BS only knows distance information of all users.

\section{B. Maximizing the fairness rate}

In this subsection, we consider to provide QoS guaranteed within a tolerable outage probability for the users. Specifically, we consider the problem of maximizing the worst user's received rate giving the maximum tolerable outage probability for each user $m$, which can be formulated as

$$
\begin{aligned}
\max _{\left\{P_{m}\right\},\left\{\tilde{R}_{m}\right\}} & \min _{m=1, \cdots, M} \tilde{R}_{m} \\
\text { s.t. } & P\left(R_{1}^{m} \geq \tilde{R}_{1}, \cdots, R_{m}^{m} \geq \tilde{R}_{m}\right) \geq 1-\epsilon_{m}, \forall m, \\
& \sum_{m=1}^{M} P_{m} \leq P, \\
& P_{m} \geq 0, \quad \forall m .
\end{aligned}
$$

With the outage constraint in (13b), by using the steps similar to those used in (5) to obtain the following relation:

$$
\phi_{m} \leq \frac{\tau_{m}^{*} P_{m}}{\sigma^{2}+\sum_{j=m+1}^{M} P_{j}}, \quad \forall m,
$$

where $\tau_{m}^{*}=\min _{j=m, \cdots, M} \frac{\log \left(1-\epsilon_{j}\right)}{\lambda_{j}}$ and $\phi_{m}$ has been defined in (3). By introducing an additional variable $t$, the optimization problem can be reformulated as

$$
\begin{array}{rl}
\max _{\left\{\left\{P_{m}\right\},\left\{\phi_{m}\right\}, t\right\}} & t \\
\text { s.t. } t & t=\min _{m=1, \cdots, M} \phi_{m}, \\
& \phi_{m}\left(\sigma^{2}+\sum_{j=m+1}^{M} P_{j}\right) \leq \tau_{m}^{*} P_{m}, \quad \forall m,
\end{array}
$$$$
\text { (13c) \& (13d). }
$$

From the definition of $\phi_{m}$, it is easy to know that $\phi_{m}>$ $0, \forall m$, is always true, which implies $t>0$ in (15). 


$$
\begin{gathered}
P_{m}=\left\{\begin{array}{l}
x_{m}+\phi_{m}\left(x_{m+1}+\sum_{k=2}^{M-m} \prod_{j=1}^{k-1}\left(1+\phi_{m+j}\right) x_{m+k}\right), m=1, \cdots, M-1 ; \\
x_{M}, m=M .
\end{array}\right. \\
\sum_{m=1}^{M} P_{m}=\sum_{m=1}^{M} \beta_{m} x_{m}, \text { where } \beta_{m}=\left\{\begin{array}{l}
1, m=1 ; \\
\prod_{i=1}^{m-1}\left(1+\phi_{i}\right), m=2, \cdots, M-1 .
\end{array}\right.
\end{gathered}
$$

Proposition 3: The optimal solution of (15) satisfies the constraints in (15c) and (13c) with strictly equality and the constraint in (13d) with strictly inequality. In addition, for (15b) the condition $\phi_{1}=\cdots=\phi_{M}=t$ will be met at the optimal solution.

Proof: See proof A.

By applying proposition 3 and using some manipulations similar to the procedures as shown in the proof of Proposition 1, we can obtain the following equations:

$$
\begin{aligned}
P_{m} & =\sigma^{2} t \sum_{j=0}^{M-m} \frac{\sum_{i=0}^{j-1}\left(\begin{array}{c}
j-1 \\
i
\end{array}\right) t^{j+1}}{\tau_{m+j}^{*}}, \\
P & =\sigma^{2} t \sum_{m=1}^{M} \frac{(1+t)^{(m-1)}}{\tau_{m}^{*}} .
\end{aligned}
$$

It can be observed that (16b) is a non-linear equation, but it can be solved by Newton's method. Then, by substituting $t$ into (16a), we can calculate individual $P_{m}, \forall m$.

IV. SOME Discussions BETWEEN NOMA AND OMA

To compare the total transmit power between NOMA and OMA from the perspective of outage probability constraints, the total transmit power is calculated by using (4) in NOMA and OMA systems separately.

From (7), the total power satisfied the outage constraint in NOMA systems can be calculated as

$$
P=\sum_{m=1}^{M} \prod_{i=1}^{m-1}\left(1+\phi_{i}\right) \phi_{m} \max _{j=m, \cdots, M} \frac{\lambda_{j} \sigma^{2}}{\log \frac{1}{1-\epsilon_{j}}}
$$

where we can observe that the total transmit power of NOMA has a linear relationship with $\sigma^{2}$.

For comparison, we introduce OMA as a comparable scheme, which can support the following data rate under the same configuration. Note that the conventional OMA system, such as TDMA, requires $M$ time slots to support $M$ users, while NOMA can support $M$ users during a single time slot. Thus, the achievable rate of OMA is

$$
\bar{R}_{m}=\frac{1}{M} \log \left(1+\frac{\left|h_{m}\right|^{2} \bar{P}_{m}}{\sigma^{2}}\right) .
$$

Similar to (3), the outage probability of OMA can be obtained. Note that the outage probability between each user in OMA is decoupled; hence, all the outage constraints must be equal at the optimal solution. Further, the total transmit power is given by

$$
\bar{P}=\sum_{m=1}^{M} \phi_{m} \frac{\lambda_{m} \sigma^{2}}{\log \frac{1}{1-\epsilon_{m}}} .
$$

Therefore, the power difference between NOMA and OMA can be calculated as (20). To be more specific, we express the power difference by logarithm,

$$
\begin{aligned}
d_{\text {gap }} & =\log \left(\frac{\sum_{m=1}^{M} \prod_{i=1}^{m-1} \omega_{i}\left(\omega_{m}-1\right) \max _{j=m, \cdots, M} \frac{\lambda_{j}}{\log \frac{1}{\tau_{j}}}}{\sum_{m=1}^{M}\left(\omega_{m}^{M}-1\right) \frac{\lambda_{m}}{\log \frac{1}{1-\epsilon_{m}}}}\right) \\
& \geq \log \left(\frac{\sum_{m=1}^{M} \prod_{i=1}^{m-1} \omega_{i}\left(\omega_{m}-1\right) \frac{\lambda_{m}}{\log \frac{1}{1-\epsilon_{m}}}}{\sum_{m=1}^{M}\left(\omega_{m}^{M}-1\right) \frac{\lambda_{m}}{\log \frac{1}{1-\epsilon_{m}}}}\right) \geq 0 .
\end{aligned}
$$

where $\omega_{m}=\phi_{m}+1$ for simplicity. It is clear that the gap is always positive, which indicates that the NOMA scheme is superior to OMA from the perspective of transmit power. Furthermore, from (20), it is easy to observe that the gap will be enhanced by increasing the number of users $M$ and the parameter $\omega_{m}, \forall m$.

\section{Simulation Results}

In this section, we present the simulation results of the proposed power allocation algorithm for NOMA systems by using only the average CSI at the transmitter. For simulation, we assume that all simulation configurations follow the system model introduced in Section II with $\gamma=2$. The distance set from the users to the $\mathrm{BS}, \mathcal{D}=\left\{d_{1}, \cdots, d_{M}\right\}$, can be generated by an arithmetic sequence. Here, it is assumed that $\mathcal{D}=\left\{d_{m} \mid d_{m}=M-m+1, \forall m\right\}$ for simplicity. Further, we suppose that all users have the same maximum outage constraint $\epsilon_{1}=\cdots=\epsilon_{M}=\epsilon$, and the same target rate $\tilde{R}_{1}=\cdots=\tilde{R}_{M}=R_{t h}$ for convenience.

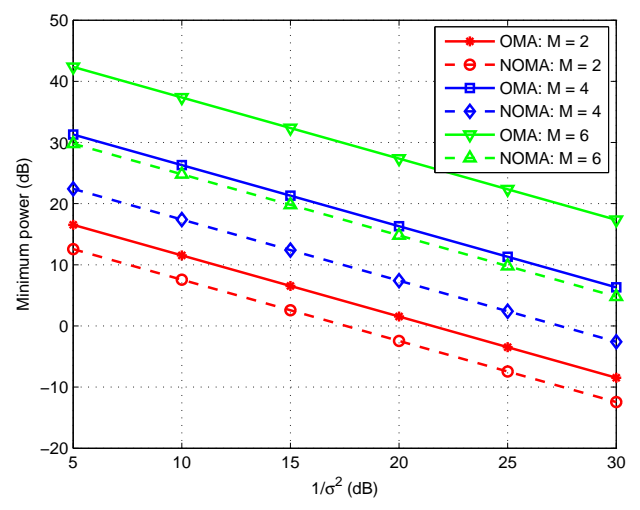

(a) Total transmission power versus $\frac{1}{\sigma^{2}}$ and $M$ with $R_{t h}=1$.

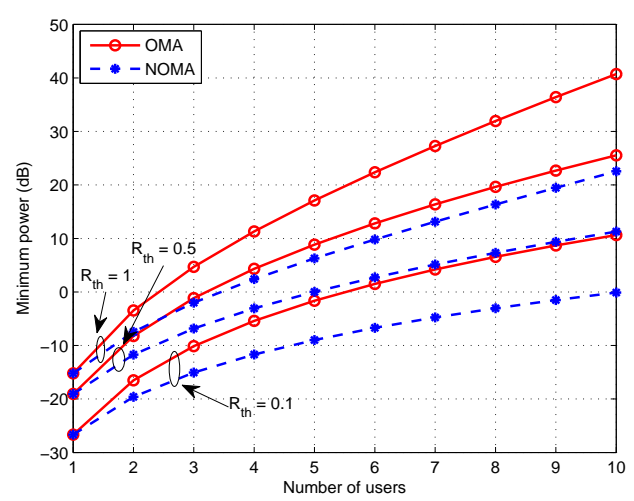

(b) Total transmission power versus $M$ and $R_{t h}$, with $\frac{1}{\sigma^{2}}=$ $25 \mathrm{~dB}$.

Fig. 1. Minimum total transmit power comparisons between downlink NOMA systems and OMA systems where $\epsilon=0.1$.

Fig. 1 demonstrates the required minimum transmit power both in NOMA systems and OMA systems separately. In Fig. 
1(a), the minimum transmit power is shown as a function of $\frac{1}{\sigma^{2}}$, where the targeted rate for NOMA is $\sum_{m=1}^{M} \tilde{R}_{m}$. It can be observed from Fig. 1(a), the gap between NOMA and OMA becomes larger with increasing $M$. Another important observation is that the total transmit power has a linear relationship with $\frac{1}{\sigma^{2}}$ in $\mathrm{dB}$, which can be derived from (17) in Section IV by some logarithmic transformations. Moreover, the impact of the number of users on NOMA and OMA is demonstrated in Fig. 1(b). From Fig. 1(b), the total transmit power will increase in order to guarantee the outage constraint since the number of the connected users is increased. However, the OMA will need more power to serve the same number of users. For example, when the transmit power is $0 \mathrm{~dB}$ and $R_{t h}=0.5$, NOMA can support 5 users while OMA can support 3 users only. It is worth pointing out that, with increasing the number of users the gap becomes a constant. Note also that as $R_{t h}$ increases, the slope of the curves changes more quickly because it is more difficult to ensure users with poor connections to be connected.

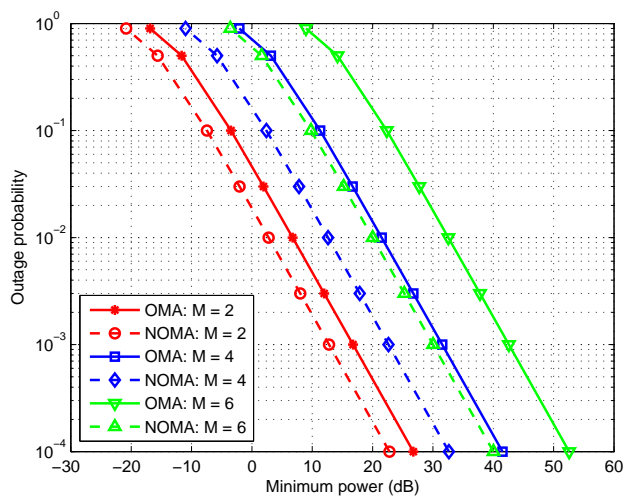

Fig. 2. Outage probility versus total transmission power and the number of users $M$ with $\tilde{R}=1$ and $\frac{1}{\sigma^{2}}=25 \mathrm{~dB}$.

Fig. 2 provides a comparison between NOMA and OMA by depicting the achievable outage probability for different number of users with varying minimum transmit power. It can be seen from Fig. 2 that NOMA and OMA can achieve the same diversity order, but the outage performance of NOMA is always better than that of OMA. In fact, as the number of users increases, the performance gain of NOMA over OMA increases almost logarithmically. In Fig. 3, the impact of the total transmit power and the user number on the maximum fairness rate of NOMA and OMA can be observed. Clearly, NOMA always outperforms OMA. In addition, the minimum rate will be obtained with increasing the user number.

\section{CONCLUSIONS}

In this letter, the problem of optimal power allocation when the transmitter only has average CSI has been studied in downlink NOMA systems. Firstly, the problem to minimize the transmit power and maximizing the fairness rate subjected to the rate outage constraint is investigated. Furthermore, the power difference between NOMA and OMA is derived. The main results of this work show that NOMA can decrease the transmit power and enhance the fairness rate by careful power allocation compared with OMA and is a promising candidate technology in future fifth generation systems.

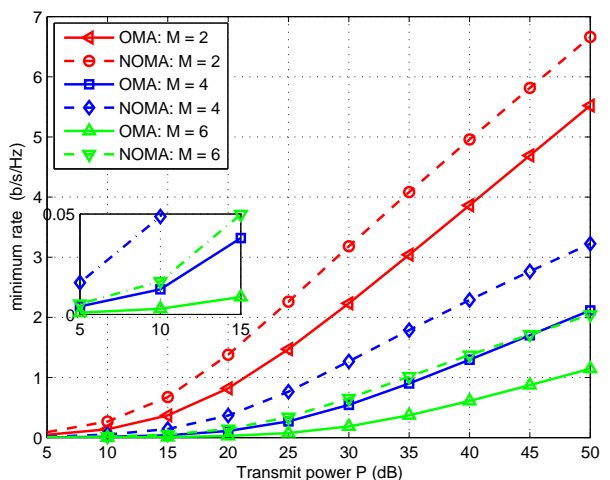

Fig. 3. The fairness rate performence comparison versus the total transmit power under different user numbers. Assume that $\frac{1}{\sigma^{2}}=1 \mathrm{~dB}$ and $\epsilon=0.1$.

\section{APPENDIX}

By replacing $t$ with $-t$ in the objective function and (15b) with $-t=\max _{m=1, \cdots, M}\left\{-\phi_{m}\right\}$, the optimization problem in (15) becomes convex. Therefore, the following Karush-KuhnTucker (KKT) conditions [8] are necessary and sufficient for optimality of (15):

$$
\begin{aligned}
& \sum_{i<m} \lambda_{i} \phi_{i}+\nu=\lambda_{m} \tau_{m}^{*}+\mu_{m}, \\
& \phi_{m}\left(\sigma^{2}+\sum_{j=m+1}^{M} P_{j}\right) \leq \tau_{m}^{*} P_{m}, \quad \forall m, \\
& \lambda_{m}\left(\phi_{m}\left(\sigma^{2}+\sum_{j=m+1}^{M} P_{j}\right)-\tau_{m}^{*} P_{m}\right)=0, \quad \forall m, \\
& \sum_{m=1}^{M} P_{m} \leq P \\
& \nu\left(\sum_{m=1}^{M} P_{m}-P\right)=0 \\
& \mu_{m} P_{m}=0 \\
& P_{m} \geq 0, \lambda_{m} \geq 0, \nu \geq 0, \mu_{m} \geq 0 .
\end{aligned}
$$

where $\lambda_{m}, \mu_{m}, \forall m$, and $\nu$ are the Lagrange multipliers for constraints (15c), (13d) and (13c) respectively. The right hand side of (21b) is strictly positive for all $m$ as $\sigma^{2}>0, \phi_{m}>0$ and $P_{m} \geq 0$; hence, the left hand side has to be strictly positive which implies that the optimal $P_{m}^{*}>0$ and $\mu_{m}^{*}=0$ (from (21f)).

Now we show that the optimal $\nu^{*}>0$ and $\lambda_{m}^{*}>0$, $\forall m$, by contradiction. If $\nu^{*}=0$, from (21a), it follows that $\lambda_{m}^{*}=\frac{\sum_{i<m} \lambda_{i} \phi_{i}}{\tau_{m}^{*}}$, which implies $\lambda_{m}^{*}=0, \forall m$. Obviously, this assumption contradicts with strong duality - Slater's condition [8]. Therefore, $\nu^{*}>0$, it follows that $\lambda_{m}^{*}=\frac{\sum_{i<m} \lambda_{i} \phi_{i}+\nu}{\tau_{m}^{*}}>$ 0.

With $\lambda_{m}^{*}>0, \nu^{*}>0$ and $\mu_{m}^{*}=0$ as proved above, conditions in (21c), (21e) and (21g) imply that all constraints in (15c) and (13c) must be enforced with equality and in (13d) with strictly inequality.

Finally, we show that $\phi_{1}^{*}=\cdots=\phi_{M}^{*}=t^{*}$ at the optimal solution. From the above proofs, (15) can be formulated as an equality constrained minimization problem. Furthermore, note that $\phi_{m}$ is not a function of $P_{j}, j<m$ and $\phi_{m}$ is monotonically increasing with $P_{m}$ and monotonically decreasing with $P_{j}, j>m$. Assume that $t=\phi_{m^{\prime}}$ with existing $\phi_{m^{\prime}} \leq \phi_{m_{1}}$, $m_{1} \neq m^{\prime}$. If $m_{1}>m^{\prime}, \phi_{m_{1}}$ has no relationship with $P_{m^{\prime}}$. At this time, there exists an $t_{1}>t$ can be attained by decreasing $P_{m_{1}}$ and increasing $P_{m^{\prime}}$ under the total power constraint. Otherwise, if $m_{1}<m^{\prime}$, we can also find an $t_{1}>t$ by similar operations under the total power constraint. Combining the two cases above, one can conclude that $\phi_{m}^{*}=t^{*}, \forall m$. Proposition 3 is thus proved. 


\section{REFERENCES}

[1] Y. Saito, Y. Kishiyama, A. Benjebbour, T. Nakamura, A. Li, and K. Higuchi, "Non-orthogonal multiple access (NOMA) for cellular future radio access," in Proc. IEEE VTC, Jun. 2013, pp. 1-5.

[2] S. Tomida and K. Higuchi, "Non-orthogonal access with SIC in cellular downlink for user fairness enhancement," in Proc. IEEE ISPACS, Dec. 2011, pp. 1-6.

[3] Z. Ding, Z. Yang, P. Fan, and H. Poor, "On the performance of nonorthogonal multiple access in $5 \mathrm{G}$ systems with randomly deployed users," IEEE Signal Process. Lett., vol. 21, no. 12, pp. 1501-1505, Dec. 2014.

[4] Z. Ding, P. Fan, and H. V. Poor, "Impact of user pairing on 5G non-orthogonal multiple access," Dec. 2014. [Online]. Available: http://arxiv.org/abs/1412.2799

[5] S. Timotheou and I. Krikidis, "Fairness for non-orthogonal multiple access in 5G systems," IEEE Signal Process. Lett., vol. 22, no. 10, pp. $1647-1651$, Oct. 2015

[6] D. Love, R. Heath, V. Lau, D. Gesbert, B. Rao, and M. Andrews, "An overview of limited feedback in wireless communication systems," IEEE J. Sel. Areas Commun., vol. 26, no. 8, pp. 1341-1365, Oct. 2008.

[7] S. Shi, L. Yang, and H. Zhu, "Outage balancing in downlink nonorthogonal multiple access with statistical channel state information," 2015. [Online]. Available: http://arxiv.org/abs/1507.04464

[8] S. Boyd and L. Vandenberghe, Convex optimization. Cambridge university press, 2004. 